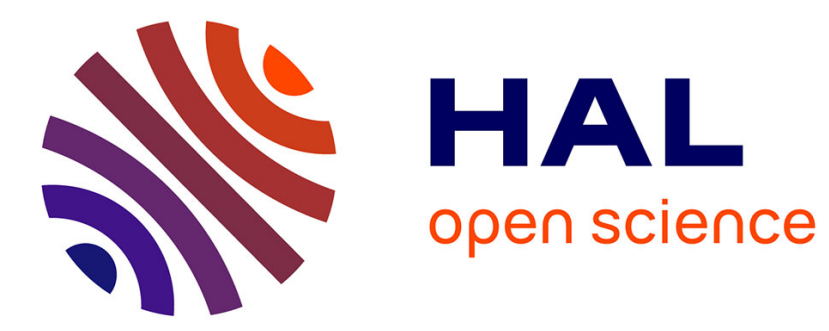

\title{
Thermoremanent Magnetization of Fine Powders
}

Louis Néel

\section{To cite this version:}

Louis Néel. Thermoremanent Magnetization of Fine Powders. Reviews of Modern Physics, 1953, 25 (1), pp.293-295. 10.1103/RevModPhys.25.293 . hal-02888377

\section{HAL Id: hal-02888377 https://hal.science/hal-02888377}

Submitted on 29 Jul 2020

HAL is a multi-disciplinary open access archive for the deposit and dissemination of scientific research documents, whether they are published or not. The documents may come from teaching and research institutions in France or abroad, or from public or private research centers.
L'archive ouverte pluridisciplinaire HAL, est destinée au dépôt et à la diffusion de documents scientifiques de niveau recherche, publiés ou non, émanant des établissements d'enseignement et de recherche français ou étrangers, des laboratoires publics ou privés. 


\title{
Thermoremanent Magnetization of Fine Powders
}

\author{
LOUIS NÉEL \\ Institut Fourier, Grenoble, France
}

\begin{abstract}
This is a brief explanation of the magnetic properties of fine ferromagnetic grains of single elementary domains, especially in reference to the influence of time on the magnetization at a given temperature. Application is made to the interpretation of the thermoremanent magnetization of lavas and clays and to the determination of the direction and intensity of the terrestrial magnetic field in the past. It is shown that, when the substance contains two kinds of fine ferromagnetic grains with clearly different Curie points, it is possible, under certain conditions explained in the text, that the constituent having the lowest Curie point becomes magnetized in a direction opposite to the exterior field: there arise, by this phenomenon, interactions between the grains and the demagnetizing field created by the constituent having the highest Curie point. Also, in certain cases, the total thermoremanent magnetization is directed inversely to the acting field. Recently Nagata found samples of lava in which the thermo-remanent magnetization obeys the mechanism thus described. In the geophysical interpretation, there will be, then, reason to keep in mind the possibility of this curious phenomenon, in considering the possible inversions of the terrestrial magnetic field.
\end{abstract}

$\mathrm{W}^{\mathrm{s}}$ $\mathrm{E}$ designate here under the name of fine powders the powders in which the grains are small enough for each one to contain only a single elementary Weiss domain. For this it is necessary that the diameter of the grains be less than a certain critical value $d_{s}$. This can be evaluated by determining the diameter of a single domain of uniform spontaneous magnetization that possesses the same free energy as a closed configuration having a resultant spontaneous magnetization equal to zero. One obtains thus ${ }^{1}$ a critical value given by

$$
d_{s}{ }^{2}=\frac{3 N a^{2}}{2 \pi}\left(\log \frac{d_{s}}{a}-0.307\right)
$$

where $N$ is the coefficient of the molecular field (5890 for iron) and $a$ the distance between magnetically active neighbors (2.86A for iron). Thus one finds that the grains of iron of a diameter less than $d_{s}=320 \mathrm{~A}$ should contain only a single elementary domain. The variations of magnetization of a group of such fine grains arise then only from the rotations of the magnetic moments resulting from the individual grains.

In the theory of their magnetic properties ${ }^{2-4}$ one takes account usually only of the rotations imposed by the magnetic field applied against the different forces of anisotropy: anisotropy of form, magnetocrystalline anisotropy, etc. . . , but in reality the grains are already so small that it is necessary to consider the spontaneous rotations due to the thermal fluctuations which provoke a kind of magnetic Brownian movement, ${ }^{4,5}$ In a group of identical grains one can define the time of relaxation $\tau_{0}$ such that, $J_{0}$ being the remanent magnetization of the group of grains at the initial instant, the magnetization at the instant $t$ is spontaneously reduced in zero exterior field to the value $J=J_{0} e^{-t / \tau 0}$.

\footnotetext{
1 L. Néel, Compt. rend. 224, 1488 (1947).

2 L. Néel, Compt. rend. 224, 1550 (1947).

${ }^{3}$ L. Weil, J. phys. et radium 12, 437 (1951).

${ }^{4}$ L. Néel, Ann. Géophys. 5, 99 (1949).
}

5 L. Néel, Compt. rend. 228, 664 (1949).
Approximate evaluation of this type of relaxation gives the result ${ }^{4,5}$

$$
\frac{1}{\tau_{0}}=\frac{e H_{c}}{m}\left|3 G \lambda_{s}+D J_{s}{ }^{2}\right|\left[\frac{2 v}{\pi G k T}\right]^{\frac{1}{2}} \exp -\frac{v H_{c} J_{s}}{2 k T},
$$

where $e$ and $m$ are the charge and the mass of electron, $k$ the Boltzmann constant, $v$ the volume of the grain, $G$ its modulus of elasticity in shear, $J_{s}$ its spontaneous magnetization, $\lambda_{s}$ its longitudinal magnetostriction at saturation, and $D$ a numerical coefficient which depends a little on the form of the grain and which is around 3. Finally $H_{c}$ is the coercive force of the group of grains in question if the phenomenon of spontaneous demagnetization did not exist. Table I gives the variations of the times of relaxation as a function of $v / T$, for iron.

This table shows at what point the time of relaxation varies rapidly with the diameter of the grains. In order that the remanent magnetization of a group of grains remain perfectly stable, it is necessary that their time of relaxation be at least equal to $10^{9} \mathrm{sec}$. Equation (1) shows that the diameter of the supposed spherical grain must then be at least equal to a certain critical diameter $d_{i}=160 \mathrm{~A}$. This discussion shows that the diameter of the grains of iron powder, used in the manufacture of permanent magnets, must lie within well determined limits. Experimental results of Bertaut confirm this point of view. ${ }^{6}$

Let us suppose now that we are concerned with a group of grains that are perfectly stable at ordinary temperature, that is, such that their time of relaxation $\tau_{0}$ is practically infinite. In order to produce a substan-

TABLE I.

\begin{tabular}{llllllll}
\hline \hline$\tau_{0}$ & $10^{-1}$ & 10 & $10^{3}$ & $10^{5}$ & $10^{7}$ & $10^{9}$ & $\mathrm{sec}$ \\
$v / T$ & 3.2 & 3.9 & 4.7 & 5.4 & 6.2 & 7.0 & $10^{-21} \mathrm{~cm}^{3} /$ degree \\
\hline \hline
\end{tabular}

${ }^{6}$ F. Bertaut, Compt. rend. 229, 417 (1949). 
tial remanent magnetization in this group, it is necessary to apply a magnetic field of the same magnitude as the coercive force $H_{c}$. As shown in Eq. (2) it is the exponential term which determines the variations of $\tau_{0}$. This time of relaxation diminishes at the same time as the factor $H_{c} J_{s} / T$ which appears in the exponential: this factor tends toward zero at the Curie point because $J_{s}$ approaches zero and $H_{c}$ diminishes when the temperature rises. Thus there exists a certain temperature $T_{b}$, lower than the Curie point called the blocking temperature, at which the time of relaxation has the order of magnitude of the duration of an experiment, several seconds to several minutes. Above this temperature, magnetization of a group of grains follows in a reversible and practically instantaneous manner the variations of the applied field and there is no more hysteresis. This blocking temperature depends on the nature and the dimensions of the grains.

At the blocking temperature and in the magnetic field $H_{b}$ the magnetic moment resulting from a group of $N$ identical grains is

$$
M_{b}=N v J_{b} \tanh \left(v J_{b} H_{b} / k T_{b}\right),
$$

where $J_{b}$ represents the value of the spontaneous magnetization of the grain at the blocking temperature. This expression results from an equilibrium, according to Lenz's law, between two possible orientations, antiparallel to each other, of the magnetic moment $v J_{b}$ of the grain. This equilibrium is defined by the value of the hyperbolic tangent, $\tanh \left(J_{b} H_{b} / k T_{b}\right)$.

Now if one lowers the temperature below $T_{b}$ while keeping the magnetic field, at least at the beginning, at the same value $H_{b}$, the time of relaxation becomes rapidly very big and the passage from one orientation of equilibrium to another becomes practically impossible. The magnetic moment of each grain preserves the orientation that it had at the temperature $T_{b}$. In other words the hyperbolic tangent maintains the same value. The magnetic moment resulting from the $N$ grains continues meanwhile to vary because of the thermal variation of the spontaneous magnetization. At the temperature $T_{0}$, lower than $T_{b}$, at which the spontaneous magnetization possesses the value $J_{0}$, this resulting moment $M_{0}$ will be given by

$$
M_{0}=N v J_{0} \tanh \left(v J_{b} H_{b} / k T_{b}\right) .
$$

The discussion of this formula shows that if $v$ is big enough, it is possible to communicate in this manner to the grains a resulting moment which is of the order of size of the saturation moment $N v J_{0}$, using a field $H_{b}$ much lower than the coercive field $H_{\mathbf{c}}$. The magnetization thus communicated is known under the name of thermo-remanent magnetization, in order to distinguish it from the remanent magnetization measured ordinarily at ordinary temperature and called isothermic remanent magnetization. Thermo-remanent magnetization has a large stability because, in order to modify its value, it is necessary to apply magnetic fields of the order of magnitude of $H_{\mathfrak{c}}$, that is, much larger than the field $H_{b}$ which gave rise to it at the temperature $T_{b}$. Finally, as long as the acting magnetic fields remain small compared with the coercive field $H_{\mathfrak{c}}$, the magnetization of the group of grains remains completely fixed in magnitude and in orientation by the field $H_{b}$, which acts on it at the moment when, for the last time, its temperature passes through the value $T_{b}$ of blocking. In order to modify the magnetization of this group in a weak field, it is necessary to heat it to the temperature $T_{b}$.

These considerations have permitted us to explain ${ }^{4}$ theoretically why the clays and lavas preserve for an indefinite time a remanent magnetization parallel to the direction of the terrestrial magnetic field that acted on them during cooling.

In a group of heterogeneous grains there exists a whole series of blocking temperatures extending from ordinary temperatures to the highest Curie point of constituents. The result is that if the magnetic field has varied during cooling the group of grains is able to register the history of this variation in a sensitive manner and to preserve it indefinitely at ordinary temperatures. One can later find it again by a series of progressive reheatings. In an analogous fashion the curious phenomena discovered by Thellier ${ }^{7}$ and Nagata $^{8}$ have been explained.

The theory of these phenomena is relatively simple when one neglects the interactions between the grains, that is, when the grains are largely separated from each other. In particular, let us consider a group of identical ferromagnetic grains which have the form of an elongated ellipsoid of revolution and of which the anisotropy is only an anisotropy of form. Their demagnetizing coefficient along the major axis can be put equal to $4 \pi / 3$ $-n$, where $n$ varies from 0 for a sphere to $4 \pi / 3$ for a cylinder. Let us suppose also that the grains are dispersed in a cluster limited exteriorly by a sphere and oriented at random along the three mutually perpendicular directions. We will designate by $c$ the fraction of the total volume of the cluster in each one of these categories; this is a measure of the density of the grouping of the grains and varies between 0 and $\frac{1}{3}$. Let us suppose now that the field $H_{b}$, applied at the blocking temperature $T_{b}$ of the grains in question, is sufficiently weak so that the argument $v J_{b} H_{b} / k T_{b}$ of the hyperbolical tangent of Eq. (4) is small compared with one. One finds then that the average remanent magnetization of the cluster at the temperature $T_{0}$ is given by the formula

$$
\bar{J}=c R s_{b} H_{b},
$$

if one neglects the interactions between the grains and if one puts

$$
R=J_{0} / J_{b} \text { and } s_{b}=v J_{b}{ }^{2} / k T_{b} .
$$

${ }^{7}$ E. Thellier, thesis, Paris, 1938; Ann. Inst. Phys. Globe 16, 157 (1938); see also bibliography in reference 4 :

${ }^{8}$ T. Nagata, Bull. Earthquake Research Inst. 21 (March, p. 1) (1943). 
When one considers these interactions, one must write

with

$$
\bar{J}=c R S_{b} H_{b},
$$

$$
S_{b}=\frac{1-2 c}{(1-2 c / 3) / s_{b}+c n} .
$$

In the lavas to which the preceding formulas are applicable, one finds that $R$ is about $4, s_{b}$ of the order of 6 to $8, n$ about 1.7 (corresponding to an ellipsoid of elongation 1.8) while $c$ lies between 0.03 and 0.20 . Practically $S_{b}$ differs generally little from $s_{b}$; the effect of the interaction is not very important.

The formulas (5) and (6) show also that the thermoremanent magnetization is proportional to $H_{b}$ when $H_{b}$ is small. One can also show that it is parallel to the field $H_{b}$ and in the same direction, which has $a$ priori appeared perfectly obvious.

The phenomena become complicated and can become extremely interesting if the cluster considered above contains two kinds $A$ and $B$ of ferromagnetic grains possessing clearly different Curie points such that the blocking temperature $T_{a}$ of the constituent $A$ having the highest Curie point is above the Curie point of the constituent $B$. Under these conditions, at the instant in the course of cooling when the temperature has attained the Curie point of $B$, the constituent $A$ has already assumed, under the action of the applied field $H_{b}$, a thermo-remanent magnetization which is henceforth quenched and which will increase automatically during the subsequent cooling without letting itself be influenced by the variations of the existing field. The thermo-remanent magnetization of $B$ develops then under the action of a field equal to the geometric sum of the applied field $H_{b}$ and of the demagnetizing field created by the grains of $A$. It may well happen that the demagnetizing field created by $A$ is larger than $H_{b}$ so that the total field acting on $B$ is directed in the direction opposite to $H_{b}$. The constituent $B$ is then magnetized in a direction opposite to that of the applied field. It can happen also that the thermo-remanent magnetization of $B$ is greater than that of $A$ in absolute value; under these conditions the total thermo-remanent magnetization resulting from the cluster is directed in the direction opposite to the field which originated it.
If the constituents $A$ and $B$ are formed of grains of the same volume and shape, with the same value of $R$, and with the individual concentrations each one equal to $3 c$, the theory shows ${ }^{9}$ that the thermo-remanent magnetization of the group is certainly negative when the following inequality is satisfied:

$$
c n R s_{b}>\frac{2\left[1-4 c+\left(8 c^{2} / 3\right)\right]}{(1-2 c)-(2 / R)(1-3 c)} .
$$

With the values of $R, n$, and $s_{b}$ indicated above, it is only necessary that the concentration $3 c$ of each of the constituents is greater than 0.28 for this condition to fulfilled. In order that negative thermo-remanence appear, it is absolutely necessary that the grains be elongated. For spherical grains $(n=0)$, the preceding inequality cannot be satisfied.

Experiment shows that the concentrations of the ferromagnetic constituents of lavas are generally less than the limiting value of 0.28 given above. But it is not necessary, in order to obtain a negative thermo-remanent magnetization, that this be the average concentration of the ferromagnetic constituents. It is sufficient that it be obtained within the complex clusters of grains. For example, one can imagine grains formed by the segregation in two phases, having different Curie points, of a solid homogeneous solution stable at a high temperature; here the necessary large concentration is automatically realized.

To summarize, the theory shows that it is possible for certain lavas to acquire a thermo-remanent magnetization directed inversely to the field which produced it. It is thus possible that certain inversions of the terrestrial magnetic field, supposed to occur in the course of geological ages and deduced from the remanent magnetization of the lavas, is in reality due simply to the specific properties of said lavas. Recently the Japanese geophysicist Nagata discovered, ${ }^{10}$ among the discharge products of the Haruna volcano, samples of lava which assumed negative thermoremanent magnetization in the laboratory. These samples contain two ferromagnetic constituents. This discovery constitutes a good experimental verification of the proposed theory. ${ }^{11}$

\footnotetext{
${ }^{9}$ L. Néel, Ann. Géophys. 7, 90 (1951).

10 T. Nagata, Nature (London) 169, 704 (1952).

${ }^{11}$ L. Néel, Compt rend. 234, 1991 (1952).
}

\section{DISCUSSION}

JOHN W. GRAHAM, Carnegie Institution of Washington, Department of Terrestrial Magnetism, Washington, D. C.: For a number of years at the Department of Terrestrial Magnetism we have been studying the extent to which it may be possible to trace the history of the earth's magnetic field in geologic time by observations of the magnetic properties of rocks. As early as 1949 we found it necessary from our observations to propose that the so-called inverse magnetizations of some rocks should be explained by factors other than a reversal of the sense of the earth's magnetic field or shifting of continental masses relative to the earth's magnetic axis. Professor Néel's subsequent treatment of this problem is thus of considerable interest and importance to us.

This summer we made extensive field samplings and observations in some remarkably well preserved and unweathered late pre-Cambrian diabase dikes in north- 